\title{
Micronutrients Should Be Monitored in the Real Practice for Korean Inflammatory Bowel Disease Patients
}

\author{
Seong-Eun Kim \\ Department of Internal Medicine, Ewha Womans University School of Medicine, Seoul, Korea
}

See "Risk Factors for Vitamin D, Zinc, and Selenium Deficiencies in Korean Patients with Inflammatory Bowel Disease" by Yoo Min Han, et al. on page 363, Vol. 11. No. 3, 2017

Patients with inflammatory bowel disease (IBD) are at risk for a variety of nutritional deficiencies. The reasons include that decreased nutrients intake, impaired absorption, intestinal loss, or increased metabolic requirement due to various clinical conditions such as systemic inflammation. ${ }^{1}$

Micronutrients are naturally occurring compounds that are not sufficiently synthesized by humans and must be obtained from the diet. Micronutrients deficiency has been reported to occur in more than half of IBD patients. Because some micronutrients are absorbed within specific locations within the gastrointestinal tract, the activity and location of a patient's IBD determines risk for specific micronutrient deficiencies. For example, IBD at terminal ileum can cause vitamin $\mathrm{B}_{12}$ deficiency, and the disease of the proximal small intestine is likely to cause deficiencies of calcium and iron. Similarly, patients who have undergone bowel resection are also at risk for micronutrient deficiencies, and the clinical manifestation depends on the extent and location of the resected bowel.

The disease activity should not be overlooked that it also contributes to micronutrient deficiency. As well as predictable reasons in an active disease such as malabsorption, diarrhea, or decreased food intake due to abdominal pain and anorexia, hypermetabolic state by systemic inflammation or drugs to treat the active diseases can impair the micronutrients absorption. For example, glucocorticoids impair Calcium, Zinc, and phosphorus absorption, causing vitamin D resistance. Long-term total parenteral nutrition can lead to deficiencies in any micronutrient not added in sufficient quantities, but most commonly include vitamins A, D, E, zinc, copper, and selenium. ${ }^{2}$

For above reasons, micronutrient deficiencies are more com- mon in Crohn's disease with small bowel disease than in ulcerative colitis, and more in active disease than at times of remission. Although the overt symptoms of micronutrient deficiency are not usually presented, micronutrient deficiency is associated with prolonged and complicated course of disease with various degrees of clinical significance. ${ }^{1}$

The most commonly deficient micronutrients in IBD have been known as vitamin $\mathrm{B}_{12}$, folate, iron, and vitamin $\mathrm{D}$. In this article, the authors focused on the deficiencies of vitamin D, and some trace elements including zinc and selenium in IBD patients. ${ }^{3}$ The value of this study presented by the authors was that it was the first one to confirm micronutrients deficiencies in Asian IBD patients, compared to the previous studies only analyzing Western data. Although only several kinds of micronutrients was investigated in this study, it was an important work which should be applied to clinical practices for Asian IBD patients, who have been in a different environment from Western patients.

However, some points should be reviewed analytically to evaluate their clinical relevance before the conclusions were fully accepted.

First, besides showing that IBD patients have significantly lower level of vitamin D, zinc, and selenium than control, the authors suggested the risk factors for deficiencies of each micronutrient in IBD patients. However, the process seemed to be rough, and not organized due to possibilities of some confound factors. Adjustment for disease duration, activity, treatment drug, and so forth, was needed to compare Crohn's disease and ulcerative colitis as a factor affecting each micronutrient deficiency. Although young age or female was suggested as a

Correspondence to: Seong-Eun Kim

Department of Internal Medicine, Ewha Womans University School of Medicine, 52 Ewhayeodae-gil, Seodaemun-gu, Seoul 03760, Korea

Tel: +82-2-2650-2845, Fax: +82-2-2655-2076, E-mail: kimse@ewha.ac.kr pISSN 1976-2283 eISSN 2005-1212 https://doi.org/10.5009/gnl17135

@) This is an Open Access article distributed under the terms of the Creative Commons Attribution Non-Commercial License (http://creativecommons.org/licenses/by-nc/4.0) which permits unrestricted non-commercial use, distribution, and reproduction in any medium, provided the original work is properly cited. 
risk factor of a certain micronutrient deficiency, the possibility that more active diseases, longer duration or histories of bowel resection were included in young or female patients in this enrollment should be considered. Although they tried to assess the disease severity by measuring serum C-reactive protein and albumin levels instead, the criteria for dividing the subgroup are very limited in estimating the proportion of disease severity.

Second, serologic markers were measured in this study to examine the micronutrient deficiency. And the reference levels of all micronutrients were from the Western data. As the authors mentioned in this article, the incidence of vitamin D deficiency might be affected by lifestyle, such as dietary intake or sunlight exposure. ${ }^{4}$ About selenium, the reference range can be influenced by race and region, that is, the soil environment in which the individuals have lived.

We also have to consider that serum levels of zinc fluctuate and reflect recent intake due to lack of storage in the body. And another thing that makes it difficult to interpret is that very little zinc is present in the serum. ${ }^{1,2}$ Due to these limitations of measurement, the subclinical zinc deficiency is difficult to be characterized and assessed in IBD patients until prominent clinical symptoms such as acrodermatitis. Although the most frequent biomarker studied is still serum zinc level, there are several candidates of measurement such as urinary zinc excretion, erythrocyte zinc concentration, platelets zinc concentration, plasma alkaline phosphatase activity, and hair zinc concentration. ${ }^{1}$

Third, the authors compared 25-(OH)D level in IBD patients to those in healthy control using a health screened cohort in the previous other study. Because the previous study was about the association of vitamin D deficiency with incidence of type 2 diabetes in high-risk Asian subjects, and they enrolled the subjects aged 30 to 69 years with routine laboratory studies, it was unclear whether the control could truly be defined as the healthy group without bowel problems. ${ }^{5}$

In addition to discussions on the association between the micronutrients and IBD in this article, their several important roles of micronutrients supplementation need to be considered.

Vitamin D supplementation affects gut inflammation and microbiota, and has the potential to reduce risk of relapse. ${ }^{6,7}$ Zinc was previously found to be in relation with the inflammatory process by ameliorating the transmucosal leak in Crohn's disease, and by decreasing the numbers of proinflammatory cells. ${ }^{8}$ Selenium could be also associated with lowering gut inflammation. In selenium deficient mice models, the colitis was exacer- bated, and the severity of the inflammation was reduced with selenium intakes. ${ }^{9}$

In conclusion, although there are some limitations, it was a significant work to show the need for monitoring these micronutrients in the real practice for Korean IBD patients in remission state as well as active state. Further studies are needed to clarify the clinical significance of low serum levels of micronutrients considering subdivided risk factors and the role of those supplementations for IBD treatment.

\section{CONFLICTS OF INTEREST}

No potential conflict of interest relevant to this article was reported.

\section{REFERENCES}

1. Weisshof R, Chermesh I. Micronutrient deficiencies in inflammatory bowel disease. Curr Opin Clin Nutr Metab Care 2015;18:576581.

2. Hwang C, Ross V, Mahadevan U. Micronutrient deficiencies in inflammatory bowel disease: from A to zinc. Inflamm Bowel Dis 2012;18:1961-1981.

3. Han YM, Yoon H, Lim S, et al. Risk factors for vitamin D, zinc, and selenium deficiencies in Korean patients with inflammatory bowel disease. Gut Liver 2017;11:363-369.

4. Shin YH, Kim KE, Lee C, et al. High prevalence of vitamin D insufficiency or deficiency in young adolescents in Korea. Eur J Pediatr 2012;171:1475-1480

5. Lim S, Kim MJ, Choi SH, et al. Association of vitamin D deficiency with incidence of type 2 diabetes in high-risk Asian subjects. Am J Clin Nutr 2013;97:524-530.

6. Jørgensen SP, Agnholt J, Glerup H, et al. Clinical trial: vitamin D3 treatment in Crohn's disease. A randomized double-blind placebocontrolled study. Aliment Pharmacol Ther 2010;32:377-383.

7. Del Pinto R, Pietropaoli D, Chandar AK, Ferri C, Cominelli F. Association between inflammatory bowel disease and vitamin D deficiency: a systematic review and meta-analysis. Inflamm Bowel Dis 2015;21:2708-2717.

8. Sturniolo GC, Di Leo V, Ferronato A, D’Odorico A, D’Incà R. Zinc supplementation tightens "leaky gut" in Crohn's disease. Inflamm Bowel Dis 2001;7:94-98.

9. Barrett CW, Singh K, Motley AK, et al. Dietary selenium deficiency exacerbates DSS-induced epithelial injury and AOM/DSS-induced tumorigenesis. PLoS One 2013;8:e67845. 